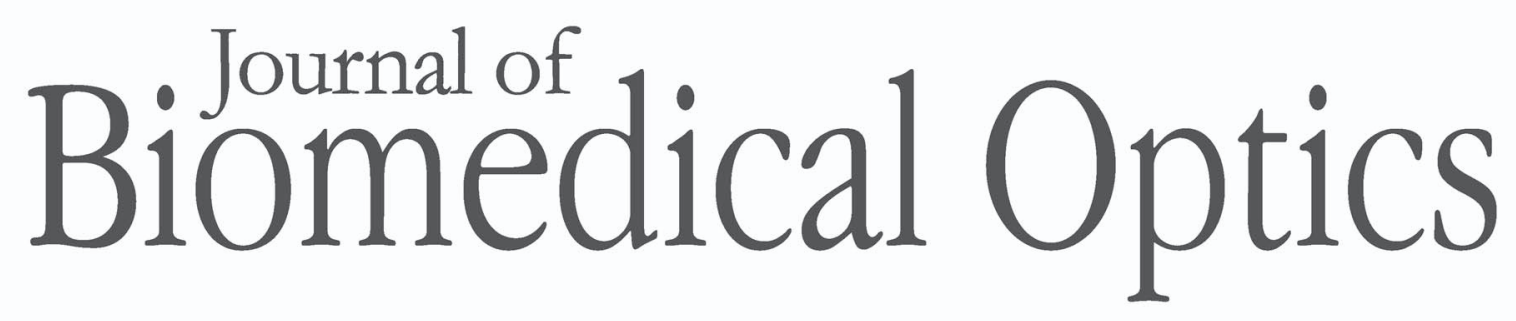

SPIEDigitalLibrary.org/jbo

\title{
Unsupervised analysis of small animal dynamic Cerenkov luminescence imaging
}

Antonello E. Spinelli

Federico Boschi 


\section{Unsupervised analysis of small animal dynamic Cerenkov luminescence imaging}

\author{
Antonello E. Spinellia and Federico Boschi ${ }^{b}$ \\ a San Raffaele Scientific Institute, Medical Physics Department, Via \\ Olgettina N. 60, Milan, Italy \\ b University of Verona, Department of Neurological, \\ Neuropsychological, Morphological and Motor Sciences, Strada Le \\ Grazie 8, 37134 Verona, Italy
}

\begin{abstract}
Clustering analysis (CA) and principal component analysis (PCA) were applied to dynamic Cerenkov luminescence images $(\mathrm{dCLI})$. In order to investigate the performances of the proposed approaches, two distinct dynamic data sets obtained by injecting mice with ${ }^{32} \mathrm{P}$-ATP and ${ }^{18}$ F-FDG were acquired using the IVIS 200 optical imager. The $k$-means clustering algorithm has been applied to $\mathrm{dCLI}$ and was implemented using interactive data language 8.1 . We show that cluster analysis allows us to obtain good agreement between the clustered and the corresponding emission regions like the bladder, the liver, and the tumor. We also show a good correspondence between the time activity curves of the different regions obtained by using CA and manual region of interest analysis on dCLIT and PCA images. We conclude that CA provides an automatic unsupervised method for the analysis of preclinical dynamic Cerenkov luminescence image data. @ 2011 Society of Photo-Optical Instrumentation Engineers (SPIE). [DOI: 10.1117/1.3663442]
\end{abstract}

Keywords: image processing; segmentation; biology.

Paper 11517LRR received Sep. 16, 2011; revised manuscript received Oct. 31, 2011; accepted for publication Nov. 2, 2011; published online Dec. 1, 2011.

Cerenkov luminescence imaging (CLI) is a new preclinical molecular imaging tool allowing studying the bio-distribution of beta emitting radiotracers ${ }^{1-7}$ using preclinical optical imaging devices.

CLI is based on the detection of visible photons emitted by charged particles, as electrons and positrons, as they travel into the tissue with energy greater than Cerenkov threshold. The basic theory behind CLI was described in Ref. 2.

The main goal of this work was to evaluate the performances of an unsupervised approach based on cluster analysis (CA) and principal component analysis (PCA) for the analysis of dynamic Cerenkov luminescence imaging (dCLI) data.

The dCLI data can be used to study the bio-distribution both spatially and temporally of radiopharmaceuticals labeled with beta plus or beta minus emitters. In this work we presented two examples using both type of radioisotopes.

In the first example attention was focused on measuring the bio-distribution of ${ }^{32} \mathrm{P}$-ATP. Such radiotracer has been widely used for in vitro biology experiments but (to our knowledge) only

Address all correspondence to: Antonello E Spinelli, San Raffaele Scientific Institute, Medical Physics Department, Via Olgettina N. 60, Milan, Italy; Tel: 0226432278; E-mail: spinelli.antonello@hsr.it.
dCLI allow investigating the spatial localization and the time course of ${ }^{32} \mathrm{P}$-ATP uptake in a mouse. As will be presented in the rest of the paper, the use of CA allows us to obtain automatically the time activity curves (TAC) of different regions like: the background, liver, bladder, etc. and, at the same time, a segmented image where all these regions are distinguishable. We believe that is a robust user independent approach to obtain the planar localization and the temporal variation of radiopharmaceuticals.

The second example included in the paper was the measurement of time activity curve of 2-[18F]fluoro-2-deoxy-D-glucose (FDG) in a tumor. Again we will show that also in this case CA allows us to correctly localize the tumor region and automatically obtain the FDG uptake curve.

CA is a well-known mathematical approach allowing to group a large data set into a smaller number of clusters having similar properties ${ }^{8}$ in our case pixels with a similar tracer kinetics. This is quite a useful tool since it allows to obtain an automatic unsupervised delineation of the different emission regions and also to extract the corresponding TAC. CA has been applied in the past to clinical ${ }^{9-11}$ and small animal dynamic positron emission tomography. ${ }^{12}$

CA can be performed by using several possible algorithms, in this work the $k$-means clustering algorithm ${ }^{13}$ has been applied by using the interactive data language ${ }^{14}$ ver. 8.1 .

Assuming that we are dealing with planar dCLI images with (pixel basis) $m$ TACs taken at $n$ different time points, the $k$-means algorithm ${ }^{13}$ is based on the minimization for $K$ clusters of the following objective function:

$$
d_{K}=\sum_{k=1}^{K} \sum_{j \in C_{k}}\left\|I_{j}-c_{k}\right\|^{2},
$$

where $c_{k}$ and $I_{j}$ correspond, respectively, to the centroid of the cluster $C_{k}$ and to the $j$ 'th TAC. More precisely, $c_{k}$ corresponds to the average of the TACs for the pixels belonging to the cluster $C_{k}$. The $k$-means clustering algorithm assigns each TAC to one cluster only.

$\mathrm{PCA}^{15}$ is a technique developed to reduce the dimensionality of a large dataset, and dimension reduction is achieved by transforming the old variables to a new set of variables called principal components. The new variables are now uncorrelated and most of the variance of the data is typically contained in the first few components. PCA has been recently applied to in vivo small animals optical imaging using fluorescent dyes. ${ }^{16}$ In Ref. 16, the time activity curves for several regions of the mouse were obtained by drawing regions of interest (ROIs) using the PCA image as a guide.

PCA analysis was performed using a routine written in MATLAB 7.1 and applied to each pixel of the images acquired at different time points. An RGB (red, blue, and green) image was then obtained by combining the first three PCA components after normalization.

In this work a comparison between TAC obtained by using CA and by manually drawing ROI respectively on the dCLI and PCA image was presented.

CLI images were acquired using the IVIS 200 optical imager (Caliper Life Sciences, Alameda, USA). The IVIS 200 is equipped with a back-thinned, back-illuminated CCD camera cooled at $-90^{\circ} \mathrm{C}$. The $\mathrm{CCD}$ has an active array of $1920 \times 1920$

1083-3668/2011/16(12)/120507/3/\$25.00 @ 2011 SPIE 


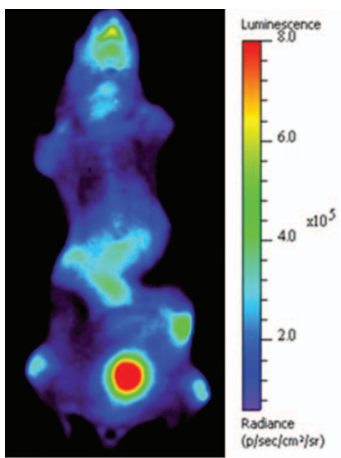

(a)

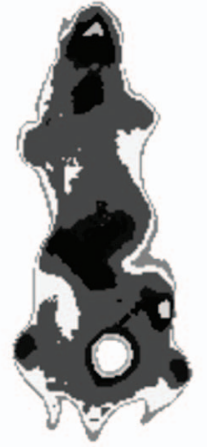

(b)

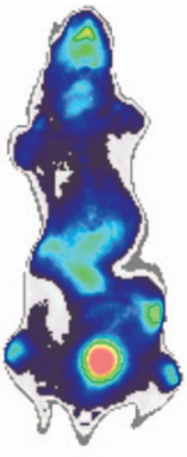

(c)
Fig. 1 (a) shows the original CLI image after $60 \mathrm{~min}$ from ${ }^{32} \mathrm{P}$-ATP tracer injection. (b) and (c) present the clustered images and the overlay of the two. As one can notice there is good agreement between the regions of the $\mathrm{CLI}$ with the corresponding regions obtained with CA.

pixels with a dimension of $13 \mu \mathrm{m}$. The images were acquired with the following parameters: exposure time equal to $300 \mathrm{~s}, f$ $=1$, binning $B=8$ and with a field of view $=12.8 \mathrm{~cm}$. The CLI data were acquired and analyzed with Living Image 4.1 (Caliper Life Sciences) and were corrected for dark measurements.

As mentioned already, in order to investigate the performances of the CA when using dCLI, two distinct data sets acquired using two different radiotracers were analyzed. The first set of dCLI was obtained by injecting a control nude mouse with $10 \pm 1 \mathrm{MBq}$ of a pure beta minus radiotracer like ${ }^{32} \mathrm{P}-\mathrm{ATP}{ }^{7}$ The second data set consisted in a BB1 tumor xenograft mouse model, in this case the animal was injected with $20 \pm 2 \mathrm{MBq}$ of a pure beta plus radiotracer like FDG.

Dynamic planar CLI scans were performed for $60 \mathrm{~min}$ after tracer injection, more precisely 10 images were acquired every 6 min.

During injection and images acquisition the mouse was kept under gaseous anesthesia ( $2 \%$ of isoflurane and $11 / \mathrm{min}$ of oxygen). All the animal handling was approved by the Institutional Ethical Committee according to the regulations of the Italian Ministry of Health and to the European Communities Council (86/609/EEC) directives.

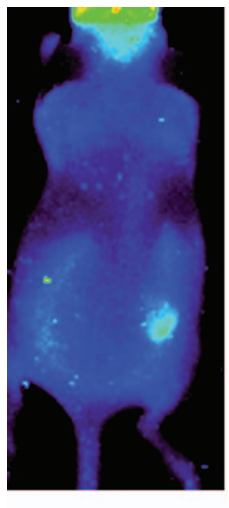

(a)

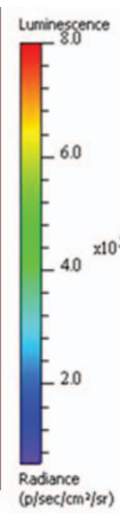

Radiance
(D/sec/cm/st)

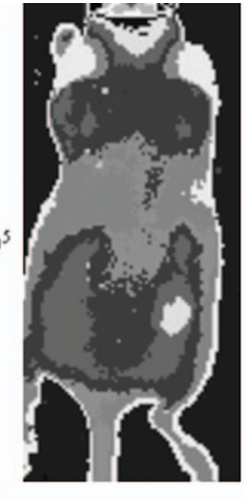

(b)

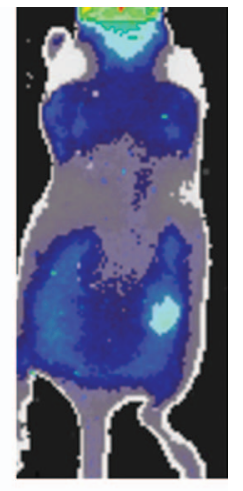

(c)
Fig. 2 (a) shows the original CLI image after 60 min from FDG tracer injection. (b) and (c) present the clustered images and the overlay of the two. It is interesting to notice that (b) shows a good delineation of the tumor region.

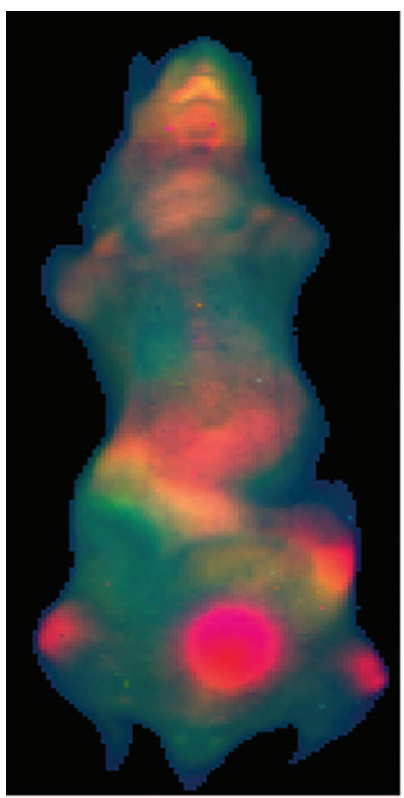

(a)

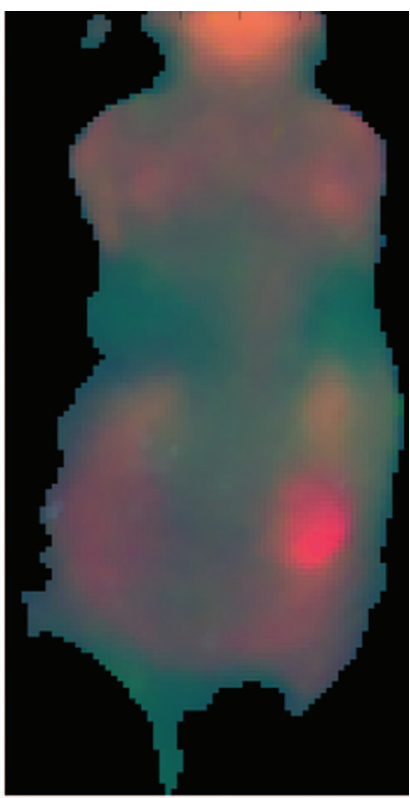

(b)
Fig. 3 The panels (a) and (b) show PCA images obtained respectively using $\mathrm{dCLI}{ }^{32} \mathrm{P}-\mathrm{ATP}$ and FDG data. The RGB images were obtained by combining the first three PCA components after normalization of each component.

Figures 1 and 2 [panel (a)] present the original CLI images obtained after $1 \mathrm{~h}$ from tracer injection and the corresponding clustered image [panel (b)]. As one can notice by looking at all the panels of Figs. 1 and 2, there is good agreement between regions such as the bladder and the liver of the CLI with the corresponding regions obtained with CA. In (b) and (c) are presented, respectively, the clustered images and the overlay of the two. In particular, Fig. 2(b) shows a good delineation of the tumor region.

As mentioned previously, the second interesting aspect of using $\mathrm{CA}$ is that it is possible to obtain directly the TAC of the different emission regions.

Figure 3 shows that the RGB image obtained by combining the first three PCA components allow a better delineation of mouse regions with different tracer kinetics. ROI analysis was performed by using $\mathrm{PCA}^{16}$ as a guide and the dCLI data in order to investigate the TAC differences with respect to CA.

In Fig. 4 are plotted the TACs of the background, liver, bladder and tumor obtained by using CA, and manual ROI drawing. It is important to notice that the plots in figure 4 show a very good agreement between the TACs of the same regions obtained with CA and manual ROI using both dCLI and PCA images as a guide. However it is useful to remind here that $\mathrm{CA}$ allows automatic TACs measurements being each TAC the centroid of each cluster.

In order to quantify the agreement between the $\mathrm{CA}$ and manual ROI a "worst case scenario" figure of merit $f$ was calculated as follows:

$$
f=\operatorname{Max}\left(\frac{\left|c_{k}-c_{\operatorname{man}}\right|}{c_{\operatorname{man}}}\right),
$$

where $c_{k}$ and $c_{\operatorname{man}}$ are, respectively, the values of the clustered and manual TACs at the different time points. The maximum value of $f$ was equal to $14 \%$ in the background region and less 


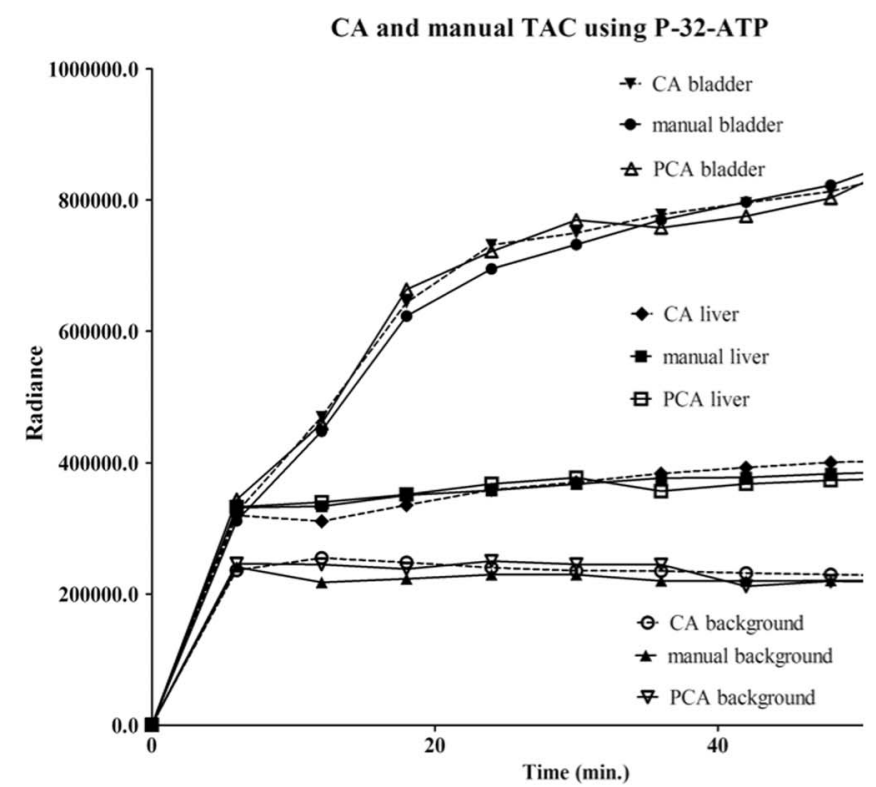

(a)

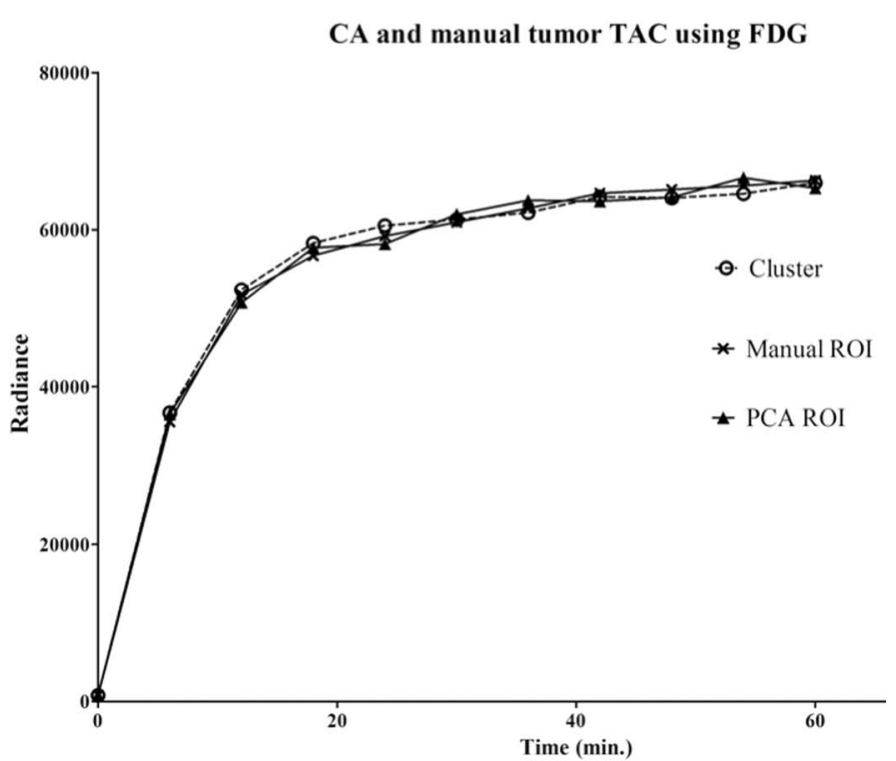

(b)

Fig. 4 The panel (a) shows the different TACs for the liver, bladder, and mouse background obtained by using CA and by manual ROI drawing on $\mathrm{dCLI}$ and PCA images. The data correspond to the control nude mouse injected with ${ }^{32} \mathrm{P}$-ATP. As one can see, the plots show very good agreement between the TACs obtained with CA and manual ROI. The $y$-axis corresponds to the photons radiance $\left(\mathrm{p} / \mathrm{s} / \mathrm{cm}^{2} / \mathrm{sr}\right)$. The panel (b) shows the TACs of the tumor region obtained using CA and by manual ROI drawing on dCLI and PCA images. The data correspond to the BB1 tumor xenograft mouse model injected with FDG.

than $8 \%$ in the bladder liver and tumor regions. These small discrepancies are well within the intrinsic experimental variability due to manual ROI dimension and position.

To summarize, the results presented in this work show that CA allows to perform unsupervised region segmentation of dCLI and to obtain the corresponding TAC. We believe that this framework will provide an easy, user independent approach for the analysis of small animal pre-clinical dCLI data.

Even if this short communication can be considered as a proof of principle, however we showed that CA performs quite well using two different tracers and mouse models. In particular the application of CA to dCLI of a xenograft mouse injected using FDG is encouraging since we show that the unsupervised CA approach can be used to obtain automatically tumor TAC.

Finally, we believe that the CA unsupervised approach can also be applied to the analyses of conventional bioluminescence and fluorescence pre-clinical optical imaging.

\section{Acknowledgments}

The authors would like to acknowledge the Cariverona Foundation and the Ospedale Sacro Cuore Don Calabria for the financial support.

\section{References}

1. R. Robertson, M. S. Germanos, C. Li, G. S. Mitchell, S. R. Cherry, and M. D. Silva, "Optical imaging of Cerenkov light generation from positron-emitting radiotracers," Phys. Med. Biol. 54, N355-N365 (2009).

2. A. E. Spinelli, D. D’Ambrosio, L. Calderan, M. Marengo, A. Sbarbati, and F. Boschi, "Cerenkov radiation allows in vivo optical imaging of positron emitting radiotracers," Phys. Med. Biol. 55, 483-495 (2010).

3. A. E. Spinelli, F. Boschi, D. D'Ambrosio, L. Calderan, M. Marengo, A. Fenzi, A. Sbarbati, A. Del Vecchio, and R. Calandrino, "Cerenkov radiation imaging of beta emitters: in vitro and in vivo results," Nucl. Instr. Meth. A 648, S310-S312 (2011).
4. F. Boschi, L. Calderan, D. D'Ambrosio, M. Marengo, A. Fenzi, R. Calandrino, A. Sbarbati, and A. E. Spinelli, "In vivo (18)F-FDG tumour uptake measurements in small animals using Cerenkov radiation," Eur. J. Nucl. Med. 38, 120-127 (2011).

5. H. Liu, G. Ren, Z. Miao, X. Zhang, X. Tang, P. Han, S. S. Gambhir, and Z. Cheng, "Molecular optical imaging with radioactive probes," PLoS One 5, e9470 (2010).

6. A. Ruggiero, J. P. Holland, J. S. Lewis, and J. Grimm, "Cerenkov luminescence imaging of medical isotopes," J. Nucl. Med. 51, 11231130 (2010).

7. A. E. Spinelli, C. Kuo, B. W. Rice, R. Calandrino, P. Marzola, A. Sbarbati, and F. Boschi, "Multispectral Cerenkov luminescence tomography for small animal optical imaging," Opt. Express 19, 12605-12618 (2011).

8. B. S. Everitt, S. Landau, and M. Leese, Cluster Analysis, John Wiley \& Sons, Ltd, Chichester, UK (2011).

9. K. P. Wong, D. Feng, S. R. Meikle, and M. J. Fulham, "Segmentation of dynamic PET images using cluster analysis," IEEE Trans. Nuclear Sci. 49, 200-207 (2002).

10. H. Guo, R. Renaut, K. Chen, and E. Reiman, "Clustering huge data sets for parametric PET imaging," BioSystems 71, 81-92 (2003).

11. M. Liptrot, K. H. Adams, L. Martiny, L. H. Pinborg, M. N. Lonsdale, N. V. Olsen, S. Holm, C. Svarer, and G. M. Knudsen, "Cluster analysis in kinetic modelling of the brain: a non-invasive alternative to arterial sampling," NeuroImage 21, 483-493 (2004).

12. S. Domenichelli, D. D'Ambrosio, S. Trespidi, C. Nanni, V. Ambrosini, S. Boschi, R. Franchi, M. Marengo, and A. E. Spinelli, "Quantitative cardiac dynamic imaging of small animal PET images using cluster analysis," Comp. in Card. 35, 337-340 (2008).

13. J. B. MacQueen, "Some methods for classification and analysis of multivariate observations," in Proceedings of the 5th Berkeley Symposium on Mathematical Statistics and Probability, Vol. 1, pp. 281-297, University of California Press, Berkeley, CA (1967).

14. http://www.ittvis.com/language/en-US/ProductsServices/IDL.aspx (last accessed 14 Oct 2011).

15. I. T. Jolliffe, Principal Components Analysis, 2nd ed, Springer-Verlag, New York (2002).

16. E. M. C. Hillman and A. Moore, "All-optical anatomical co-registration for molecular imaging of small animals using dynamic contrast," Nat. Photon. 1(9), 526-530 (2007). 